\title{
Multi-scale EBSD and EDS for Detection and Analysis of Spatially Rare Grains and Phases
}

\author{
Jenny Goulden ${ }^{1}$, Scott Sitzman ${ }^{2}$, Kim Larsen ${ }^{1}$, Hui Jiang ${ }^{1}$ \\ ${ }^{1}$ Oxford Instruments NanoAnalysis, High Wycombe, Bucks, UK \\ ${ }^{2}$ Oxford Instruments America, Concord, MA, USA
}

Analysis of sparsely distributed, minor phases and grains of interest can represent a challenge in EBSD, due to the conflicting demands of locating them over a large sample area and analyzing them at adequate grain pixilation levels. However, modern EBSD-EDS microanalysis systems, such as the Oxford Instruments AZtec system, deliver functionality that is beneficial in addressing this challenge, including a very high data acquisition rate potential, automated beam-stage large area mapping capability, handling of large datasets, EDS-EBSD data integration, and rapid, efficient diffraction pattern storage. High data collection rates allow relatively fine grid spacings for a given area of coverage and session time, increasing the potential for mapping over large specimen areas at grid resolutions sufficiently fine for analysis of intra-granular features. In addition, since the data at each gridpoint can include an EDS spectrum, EBSD indexing results and a stored diffraction pattern, location and full identity verification of revealed phases of interest are possible offline, as is large scale-tosubgrain scale EDS-EBSD analysis for all phases.

In more challenging samples, where proper indexing demands higher quality/lower speed camera settings, or where grain size is relatively small, large area coverage and fine map grid spacing may be incompatible for a given session time. In such cases, a two-staged mapping approach is needed, including an initial, large area survey map from which phases of interest can be located, even if not well resolved, followed by targeted re-location and data collection at grid resolutions commensurate with the needs of the final analysis. Like the single-dataset method described above, this method uses stored EDS, EBSD and imaging data to locate and verify the identity of phases/grains of interest, then provides a reference for stage re-positioning to these locations for higher-resolution map collection. The latter step can be performed in a later SEM session by re-loading the sample and applying image registration tools to maps and SEM images from the initial survey dataset, such that the stage may be automatically and accurately moved to a selected grain of interest.

Analysis of a bent nickel tube [1] is well served using the multi-scale approach. Large area mapping reveals mm-scale regions of similarly oriented grains (Fig. 1a) and sample-scale distributions of plastic strain (Fig. 1b), with clearly demarked regions of compressive and tensile deformation bounding an intermediate, lower deformation zone. Using the map in Fig. $1 \mathrm{~b}$ to directly reposition the stage to a location within that zone, a small area was remapped at higher spatial resolution and very high orientation accuracy using the AZtec Refined Accuracy mode. The highly detailed, subgranular deformation structure in a selected grain is shown using a local misorientation map (Fig. 1c) and a disorientation map (Fig. 1d).

The approach is also useful for characterization of terrestrial rocks and meteorites where chemically or crystallographically distinctive, but spatially rare, phases are of interest and need to be mapped at resolutions sufficient for characterization of intra-granular features.

References: 
[1] H S Ubhi, H. Jiang, Study of Recovery and Recrystallisation in Folded BCC, FCC and HCP Sheet Samples, Materials Science Forum Vols. 715-716 (2012) pp 770-775

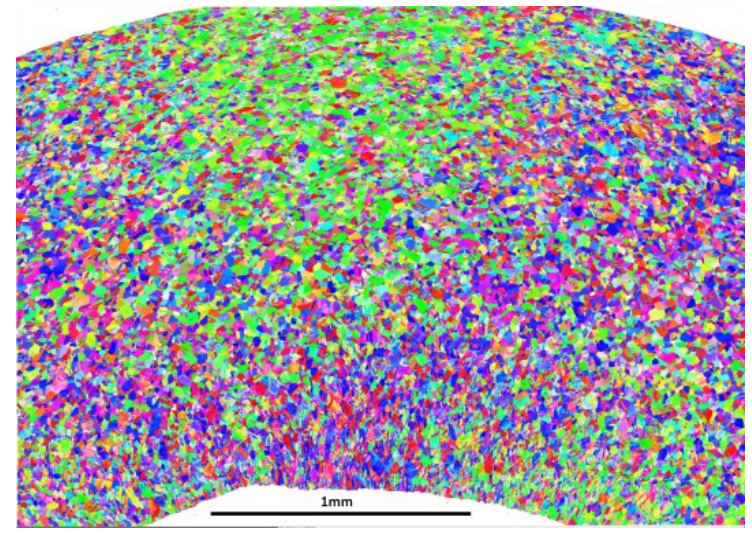

Figure 1a. Large area map of a section of Ni pipe, composed of more than 90 individual montaged fields. IPF-Y based orientation map shown.

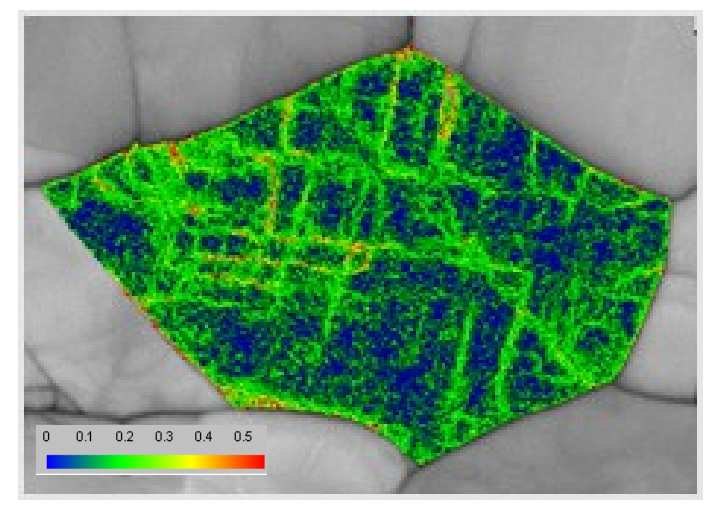

Figure 1c. A high resolution map of a single grain displayed in "local misorientation" coloring. Individual pixels are colored by misorientation with respect to neighboring pixels. A geometrical array of subtle ( $<1$ degree) subgrain boundaries is revealed. Legend values in degrees.

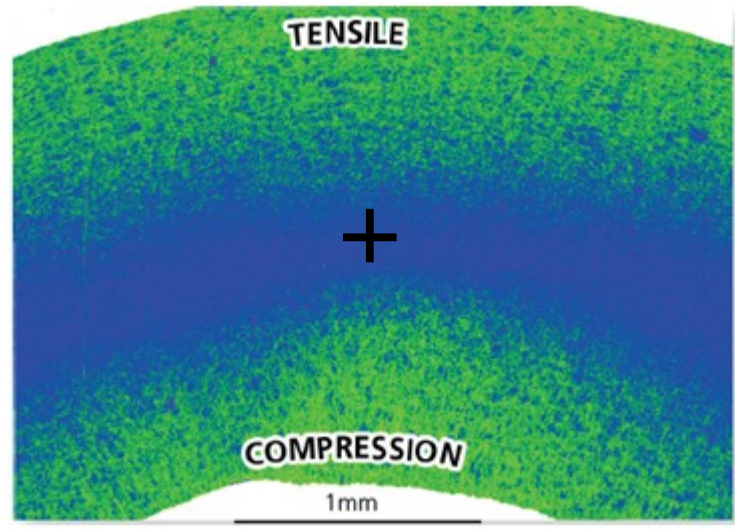

Figure 1b. Local mis-orientation map. Green regions indicate higher plastic strain, either from tension or compression. This was used to target a higher resolution map from the central, lower deformation region, denoted by the black cross.

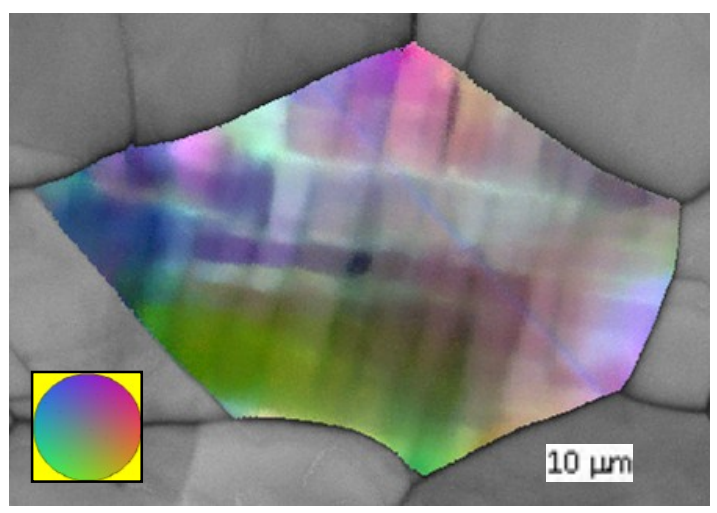

Figure 1d. "Disorientation component" map, showing internal structure by local rotation axis orientation. 